\title{
PID CONTROLLER DESIGN WITH SPECIFICATIONS ON THE INFINITY-NORM OF SENSITIVITY FUNCTIONS
}

\author{
D. Garcia, A. Karimi and R. Longchamp \\ Laboratoire d'Automatique, EPFL, CH-1015 Lausanne, \\ Switzerland. e-mail: daniel.garcia@epfl.ch
}

\begin{abstract}
A controller design method using a frequency criterion is proposed in this paper. The frequency criterion is defined as the weighted sum of squared errors between the specified and computed values of the design parameters. These are the infinity-norm of the sensitivity and complementary sensitivity functions and the crossover frequency. The criterion is then minimized iteratively using the GaussNewton method. Robustness against the model uncertainties are also investigated by including uncertainty profiles in the criterion. Simulation examples show the effectiveness and simplicity of the proposed method. Copyright $\left.{ }^{(}\right) 2005$ IFAC
\end{abstract}

Keywords: PID controllers, robust control, linear systems

\section{INTRODUCTION}

PID controllers are undeniably the most commonly used control algorithm for industrial processes. In spite of their very simple structure, they often perform well and can meet the specifications provided that their parameters are properly chosen.

The classical loop frequency specifications are habitually expressed in terms of phase margin, gain margin and crossover frequency (Ho et al., 1995). An iterative model-free method proposed in Karimi et al. (2003) is based on a frequency criterion, which is defined as the weighted sum of squared errors between the measured and desired values of these design parameters. But gain and phase margins only measure the distance in certain specific directions. If one of them is small, the closed-loop system is close to instability. However, for some systems like resonant mechanical processes, this can be the case if both margins are relatively large.

On the other hand, classical robust techniques such as $H_{\infty}$-minimization solve successfully robust design issues. Their drawback consists mainly in the fact that the obtained results are statespace controllers having the same order as the plant model, rather than low-order controllers with fixed structure. Furthermore, low order plant models containing time-delay, which are practically preferred for the tuning of controllers with simple structures, cannot be handled by such techniques.

Conversely, the proposed approach can handle as well plant models with time-delay as systems given only by a non-parametric model in the freqency domain. It considers specifications on the infinity-norm of the sensitivity and complementary sensitivity functions as well as on the crossover frequency. These specifications represent the stability and performances of the closed-loop system. The controller parameters are then obtained by minimizing a frequency criterion. A further contribution of this paper is to give the possibility of considering an unstructured uncertainty profile in the design procedure. Since many on-line identification methods for PID controller tuning produce low-order models that are not always very representative of real processes, the consideration 
of uncertainties can be strongly desired, in order to guarantee the stability of the real system.

The paper is organized as follows: The problem is formulated is Section 2, the choice of the design parameters is motivated and the frequency criterion is presented. In Section 3 the iterative solution of the problem is presented, and simulation examples are provided in Section 4. The robustness against model uncertainties are treated in Section 5. Finally some concluding remarks are offered in Section 6.

\section{PROBLEM FORMULATION}

In this paper, we aim to propose a controller design method that should be applicable to a wide range of industrial plants. The plant model is assumed to be linear and specified either by a transfer function $G(s)$ that can be of infinite order (i.e. containing a time-delay) or a non-parametric model $G(j \omega)$.

The procedure is in particular adapted to tune the parameters of PID controllers, which are the most commonly used control algorithms in industrial processes. However, it should be noted that the proposed design procedure does not make any assumption on the controller structure and is, therefore, not restricted to PID controllers. The only requirement is that an initial stabilizing controller exists prior to the design.

\subsection{Choice of Design Parameters}

The choice of design parameters plays a crucial role on the achieved performances and stability of the closed-loop system. Design parameters must ideally satisfy three principal requirements:

- They should have a universal character. In other words, the choice of specified values for the design parameters should be a priori known and should preferably not depend on the system structure and parameters. In particular, different processes with the same controller structure, designed with the same specifications, should exhibit similar behaviors, in a normalized time scale.

- The effect of specifications must be transparent to the user. In other words, it should be very intuitive which design parameter has an direct influence on a specified closed-loop system property.

- The design parameters should capture the essence of the control problem. They should reflect the stability, the robustness as well as the time-domain performances of the closedloop system. Moreover, their number have to be limited.
In the sequel, the chosen design parameters are introduced.

Modulus Margin: Let the loop transfer function be $L(s)=K(s) G(s)$, where $K(s)$ is the controller transfer function and let the sensitivity function be $S(s)=(1+L(s))^{-1}$. The sensitivity function expresses the amplification of disturbances at the output of the plant by the closed-loop system. It also quantifies how sensitive is the closedloop system to variations of the considered plant. The modulus margin $M_{m}$, which is defined as the inverse of the infinity-norm of the sensitivity function: $M_{m}=\|S(s)\|_{\infty}^{-1}$ is chosen as a design parameter for the proposed method. This value is simply the shortest distance from the Nyquist curve of the loop transfer function to the critical point -1 . It follows that the frequency response of the open-loop system, satisfying this specification, is tangent to the circle centered in $(-1 ; 0)$ with a radius of $M_{m}$. This ensures a lower bound for the gain margin of $\frac{1}{1-M_{m}}$. Furthermore it is related to the upper bound for the disturbances amplification by the closed-loop system. Typical values of $M_{m}$ are in the range of $0.5-0.75$.

Complementary Modulus Margin: Let the complementary sensitivity function be $T(s)=\frac{L(s)}{1+L(s)}$, which represents the transfer function from setpoint to process output. The second design parameter that we define as the complementary modulus margin $M_{c}$, is equal to the inverse of the infinity-norm of the complementary sensitivity function: $M_{c}=\|T(s)\|_{\infty}^{-1}$. Its value is related to the resonance peak of the transfer function from setpoint to process output and thus constitutes an important performance indicator of the response to setpoint changes. It can be shown that the frequency response of the open-loop system satisfying a given specification on $M_{c}$ will be tangent to a circle in the complex plane centered in $\left(-\frac{1}{1-M_{c}^{2}}, 0\right)$ with a radius of $\frac{M_{c}}{1-M^{2}}$. The corresponding disk defines a prohibited zone for the open-loop frequency response in the complex plane. Note that a specified value for $M_{c}$ of 1 corresponds to a prohibited disk of a radius infinitely large, and is in the proposed procedure numerically not desired. A specification on $M_{c}$ ensures a lower bound for the phase margin $\Phi_{m}$ of:

$$
\Phi_{m} \geq-\arccos \left(\frac{M_{c}^{2}-2}{2}\right)+\pi \approx 63 M_{c}-3
$$

Recommended value for the largest magnitude of the complementary sensitivity function is typically between 1.0 and 1.5, which gives a complementary modulus margin $M_{c}$ between 0.65 and 1.0. These values corresponds approximatively to overshoots of $30 \%$ and respectively $0 \%$.

Crossover Frequency: If desired by the control designer, the proposed method allows also the 
crossover frequency $\omega_{c}$ to be considered as a design parameter. $\omega_{c}$ is defined as the frequency at which the loop amplitude is one $\left(\left|L\left(j \omega_{c}\right)\right|=1\right)$. A specified value for $\omega_{c}$ is however not a priori known and depends especially on the plant dynamics. Since the crossover frequency is closely related to the rise time $\tau$ and thus to the bandwidth of the closed-loop system, it constitutes a performance indicator. Guiding rules on the choice of this specification based on the desired rise time $\tau$ can be formulated. The rule $\omega_{c}=2.3 / \tau$ gives often satisfactory results in practice. It comes from the consideration that the closed-loop system behaves roughly like a second-order system. It applies approximately and only to stable minimum-phase systems.

\subsection{Frequency Criterion}

The problem, with design parameters discussed in the previous sections, can now be formulated as an optimization problem: Find a controller that minimizes a frequency criterion, which is defined as the weighted sum of squared errors between the specified and computed values of the frequency characteristics. The criterion is defined as:

$$
\begin{aligned}
& J(\rho)=\frac{1}{2}\left(\lambda_{1}\left(M_{m}(\rho)-M_{m}^{*}\right)^{2}\right. \\
& \left.\quad+\lambda_{2}\left(M_{c}(\rho)-M_{c}^{*}\right)^{2}+\lambda_{3}\left(\omega_{c}(\rho)-\omega_{c}^{*}\right)^{2}\right)
\end{aligned}
$$

where $\rho$ is the vector of the controller parameters of dimension $n_{\rho}, \lambda_{1}, \lambda_{2}$ and $\lambda_{3}$ are weighting factors, $M_{m}$ and $M_{m}^{*}$ are respectively the actual and specified value of the modulus margin. $M_{c}$ and $M_{c}^{*}$ are the actual and desired complementary modulus margin and $\omega_{c}$ and $\omega_{c}^{*}$ the actual and desired crossover frequency. The weightings factors are usually chosen as : $\lambda_{1}=1 / M_{m}^{* 2}, \lambda_{2}=1 / M_{c}^{* 2}$ and $\lambda_{3}=1 / \omega_{c}^{* 2}$, in order to normalize the terms in the criterion. In the sequel it is assumed that the value of $M_{m}, M_{c}$ and $\omega_{c}$ can be computed numerically using the plant model and the in-hand controller transfer function.

\section{ITERATIVE SOLUTION}

The controller parameters minimizing the criterion can be obtained iteratively by the GaussNewton method:

$$
\rho_{i+1}=\rho_{i}-\gamma_{i} R^{-1} J^{\prime}\left(\rho_{i}\right)
$$

where $i$ is the iteration number, $\gamma_{i}$ the step size, $R$ a positive definite matrix of dimension $n_{\rho} \times n_{\rho}$ that can be chosen equal to the Hessian $H$ for a fast convergence and $J^{\prime}(\rho)$ is the gradient of the criterion with respect to $\rho$. Note that this iterative algorithm gives only a local minimum of the criterion, so the initial choice of controller plays an important role.
The gradient of the criterion is given by:

$$
\begin{aligned}
& J^{\prime}(\rho)=\lambda_{1}\left(M_{m}-M_{m}^{*}\right) \frac{d M_{m}}{d \rho}+ \\
& \lambda_{2}\left(M_{c}-M_{c}^{*}\right) \frac{d M_{c}}{d \rho}+\lambda_{3}\left(\omega_{c}-\omega_{c}^{*}\right) \frac{d \omega_{c}}{d \rho}
\end{aligned}
$$

and the Hessian of the criterion is given by:

$$
\begin{aligned}
& H(\rho)=\lambda_{1} \frac{d M_{m}}{d \rho}\left(\frac{d M_{m}}{d \rho}\right)^{T}+\lambda_{2} \frac{d M_{c}}{d \rho}\left(\frac{d M_{c}}{d \rho}\right)^{T} \\
& +\lambda_{3} \frac{d \omega_{c}}{d \rho}\left(\frac{d \omega_{c}}{d \rho}\right)^{T}+\lambda_{1}\left(M_{m}-M_{m}^{*}\right) \frac{d^{2} M_{m}}{d \rho^{2}} \\
& +\lambda_{2}\left(M_{c}-M_{c}^{*}\right) \frac{d^{2} M_{c}}{d \rho^{2}}+\lambda_{3}\left(\omega_{c}-\omega_{c}^{*}\right) \frac{d^{2} \omega_{c}}{d \rho^{2}}
\end{aligned}
$$

The last three terms containing the second derivatives can be neglected because they are small especially in the neighborhood of the optimum. In addition, this simplifies largely the computation of the Hessian, which can be computed without any additional information, since the remaining terms are also present in the gradient. Hence:

$$
\begin{aligned}
H \approx \lambda_{1} \frac{d M_{m}}{d \rho}\left(\frac{d M_{m}}{d \rho}\right)^{T} & +\lambda_{2} \frac{d M_{c}}{d \rho}\left(\frac{d M_{c}}{d \rho}\right)^{T} \\
& +\lambda_{3} \frac{d \omega_{c}}{d \rho}\left(\frac{d \omega_{c}}{d \rho}\right)^{T}
\end{aligned}
$$

Note that the rank of the Hessian is less or equal to the number of design parameters effectively considered (number of non-zero $\lambda_{k}, k=1 \ldots 3$ ). Then if the number of controller parameters is larger than that of the design parameters, the obtained Hessian is singular. To avoid this problem, one can either reduce the number of controller parameters (for a PID controller by fixing a constant ratio between the integral and derivative term for example), or consider a modified Hessian $\tilde{H}=H+$ $\epsilon I$, where $\epsilon$ is a small positive number.

It now remains to determine the derivatives of $M_{m}, M_{c}$ and $\omega_{c}$ with respect to the controller parameters $\rho$.

\subsection{Derivative of the Modulus Margin}

Consider $\omega_{M_{m}}$ as the frequency where the sensitivity function reaches its maximum value. $\omega_{M_{m}}$, is obviously a function of the controller parameters $\rho$. The term $M_{m}^{\prime}\left(=\frac{d M_{m}}{d \rho}\right)$ can then be computed through the chain rule as follows:

$$
M_{m}^{\prime}=\frac{\partial M_{m}}{\partial \rho}+\left.\frac{\partial M_{m}}{\partial \omega}\right|_{\omega_{M_{m}}} \frac{\partial \omega_{M_{m}}}{\partial \rho}
$$

The first term in the above equation can be written as:

$$
\frac{\partial M_{m}}{\partial \rho}=\frac{\partial\left|1+L\left(j \omega_{M_{m}}\right)\right|}{\partial \rho}
$$

Its numerical value can easily be computed, since it does not contain any derivatives of the plant model. 
The second term:

$$
\left.\frac{\partial M_{m}}{\partial \omega}\right|_{\omega_{M_{m}}}=\left.\frac{\partial|1+L(j \omega)|}{\partial \omega}\right|_{\omega_{M_{m}}}
$$

is the derivative of $|1+L(j \omega)|$ with respect to the frequency at the minimum value of this function. Since the function $|1+L(j \omega)|$ and its first derivative with respect to the frequency are continuous functions, the latter is obviously zero at the minimum value of the function. Thus one has: $\left.\frac{\partial M_{m}}{\partial \omega}\right|_{\omega_{M}}=0$, and consequently:

$$
M_{m}^{\prime}=\frac{\partial\left|1+L\left(j \omega_{M_{m}}\right)\right|}{\partial \rho}
$$

Thus, the derivative of the modulus margin with respect to the controller parameters can be computed analytically without any derivatives of the plant model, since these derivatives with respect to the controller parameters are zero. The only measurement of $G\left(j \omega_{M_{m}}\right)$ is sufficient for determining $M_{m}^{\prime}$.

\subsection{Derivatives of the Complementary Modulus Margin}

The derivative $M_{c}^{\prime}$ can be computed as:

$$
M_{c}^{\prime}=\frac{d M_{c}}{d \rho}=\frac{\partial M_{c}}{\partial \rho}=\frac{\partial\left|\frac{1+L\left(j \omega_{M_{c}}\right)}{L\left(j \omega_{M_{c}}\right)}\right|}{\partial \rho}
$$

where $\omega_{M_{c}}$ is the frequency where the magnitude of complementary sensitivity is maximal. The developments are identical to those made to compute the derivative of the modulus margin and are consequently omitted. But again, this derivative does not contain any derivatives of the plant, since its derivative with respect to the controller parameters are zero.

\subsection{Derivative of the Crossover Frequency}

To compute the derivative of the crossover frequency, we use the fact that the loop gain at $\omega_{c}$ is by definition always equal to 1 . Its derivative with respect to $\rho$ is therefore zero (Karimi et al., 2003):

$$
\begin{aligned}
& \frac{d\left|L\left(j \omega_{c}\right)\right|}{d \rho}=\frac{\partial\left|L\left(j \omega_{c}\right)\right|}{\partial \rho}+ \\
& \left.\frac{\partial|L(j \omega)|}{\partial \omega}\right|_{\omega_{c}} \frac{\partial \omega_{c}}{\partial \rho}=0
\end{aligned}
$$

Thus $d \omega_{c} / d \rho$ can be written as follows:

$$
\frac{\partial \omega_{c}}{\partial \rho}=-\frac{\partial\left|L\left(j \omega_{c}\right)\right|}{\partial \rho}\left[\left.\frac{\partial|L(j \omega)|}{\partial \omega}\right|_{\omega_{c}}\right]^{-1}
$$

The last term of Eq. 13 can be written as:

$$
\begin{array}{r}
\left.\frac{\partial|L(j \omega)|}{\partial \omega}\right|_{\omega_{c}}=\left.\left|G\left(j \omega_{c}\right)\right| \frac{\partial|K(j \omega)|}{\partial \omega}\right|_{\omega_{c}}+ \\
\left.\left|K\left(j \omega_{c}\right)\right| \frac{\partial|G(j \omega)|}{\partial \omega}\right|_{\omega_{c}}
\end{array}
$$

and thus contains a derivative of the plant with respect to the frequency. If no parametric model of the plant is available, this term can be approximated with appropriate accuracy thanks to the Bode's integrals (Karimi et al., 2003) or using numerical computations (Garcia et al., 2003).

\section{SIMULATION EXAMPLES}

Now, two different parametric plant models are considered to illustrate the proposed method:

$$
\begin{aligned}
& G_{p 1}(s)=\frac{-0.2 s+1}{(s+1)^{2}} e^{-0.1 s} \\
& G_{p 2}(s)=\frac{1}{s^{2}+1.7 s+1} e^{-0.05 s}
\end{aligned}
$$

Since it is usual to include a noise filter for the derivative term, the following controller structure is used :

$$
K(s)=K_{p}\left(1+\frac{1}{T_{i} s}+\frac{T_{d} s}{\frac{T_{d}}{20} s+1}\right)
$$

For each plant model an initial PID controller is first designed using the Kappa-Tau tuning rules (K. J. Aström and T. Hägglund, 1995). This one is an empirical method that also uses the infinitynorm of the sensitivity function as a design parameter. The proposed method is then used to adjust the robustness, stability and performances of the closed-loop system. The specifications are set for each closed-loop system to 1.40 for the infinitynorm of the sensitivity function $\left(M_{m}^{*}=0.714\right)$ and 1.03 for the infinity-norm of the complementary sensitivity function $\left(M_{c}^{*}=0.97\right)$. No value is however specified for the crossover frequency $\left(\lambda_{3}=0\right)$. A controller having only two parameters can then be sufficient to minimize the criterion and makes the Hessian strictly positive definit. The number of controller parameters are set to two by choosing the constant ratio $T_{i}=4 T_{d}$ between the integral and derivative time. It is pointed out in K. J. Aström and T. Hägglund (1995) that this ratio is appropriate for many industrial processes.

The resulting controllers are then compared with those obtained with the Kappa-Tau method and the Ziegler-Nichols tuning rules. It should be noted that the Kappa-Tau method uses the same specified value (1.40) for the infinity-norm of the sensitivity function. 


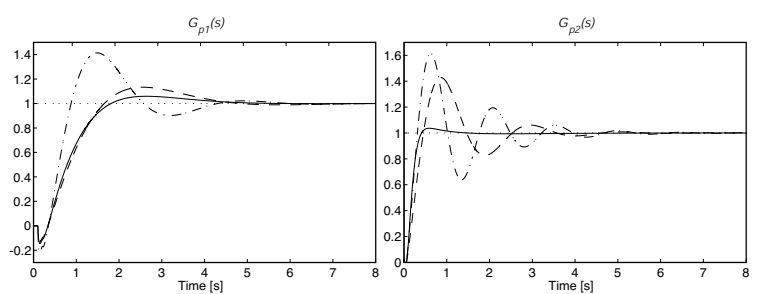

Fig. 1. Step responses (dashed-dotted: ZieglerNichols, dashed: Kappa-Tau, solid: proposed)
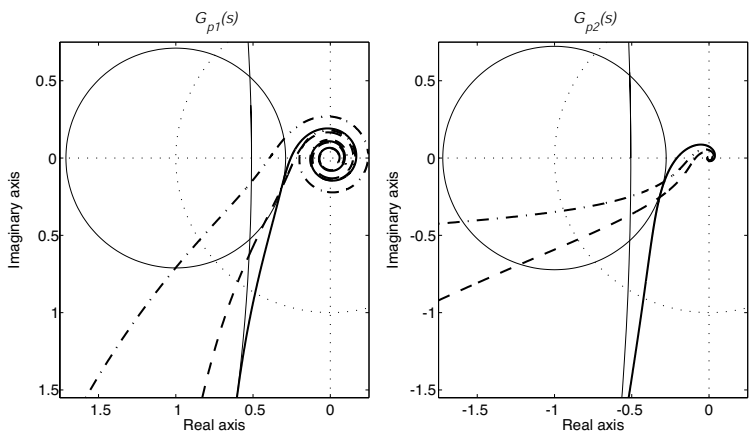

Fig. 2. Nyquist plots (dashed-dotted: ZieglerNichols, dashed: Kappa-Tau, solid: proposed)

To facilitate the comparison between the controllers, the results are given in tables and graphs. Step responses obtained in closed-loop with the different controllers are compared in Fig. 1.

The details of design, the related performances and robustness achievements are shown in Table 1, where $o$ stands for the overshoot (in \%), and $t_{s}$ for the settling time to $1 \%([\mathrm{~s}])$. These results show that the proposed controller improve considerably the time-domain performances of the closed-loop system. In each case, both settling time and overshoot have been clearly reduced. Furthermore, there is a large similarity between the responses obtained with the same specifications on the design parameters. This shows that the modulus margin and the complementary modulus margin constitute suitable design parameters.

Figure 2 shows the Nyquist plots of the loop transfer functions for each model. A circle corresponding to the specified modulus margin and a part of the circle corresponding to the specification on the complementary modulus margin are also shown in the figure. The Nyquist curves of the open-loop systems designed with the proposed method fulfill the requirement contrary to the others.

\section{STABILITY ROBUSTNESS AGAINST MODEL UNCERTAINTIES}

Since a nominal plant model represents only an approximation of the real process, modeling errors might adversely affect the robustness and stability of the control system. Therefore, it can be desired to ensure a given modulus margin to a set of models containing the real system and not only to the nominal plant model. For this purpose, plant model uncertainty profiles can be determined. The proposed controller design method can then be adapted by taking these profiles into account and guarantee a specified lower bound for the modulus margin of the real control process.

Unstructured uncertainties are practically preferred because of their generality: They can include parametric uncertainties as well as unmodeled dynamics. In particular, unstructured disk uncertainties are chosen for detailed study, because these uncertainty profiles are easy to create and their analysis is general and simple. On the other hand, the obtained solution on the real system will be conservative relative to the specifications.

Suppose first that the frequency response of a nominal stable plant transfer function is $G_{n}(j \omega)$ and that the true physical process $G_{t}(j \omega)$ belongs to a set $G$ of stable perturbed frequency responses defined as:

$$
G_{t}(j \omega) \in G=\left\{G_{n}(j \omega)(1+W(j \omega) \Delta(j \omega))\right\}
$$

The uncertainty profile $W(j \omega)$ is the frequency response of a fixed stable transfer function that gives upper bounds for the uncertainty amplitude. $\Delta(s)$ is a variable stable function satisfying $\|\Delta(s)\|_{\infty} \leqslant 1$. In this case the perturbation model is said to be multiplicative. Now it is desired to measure the minimal modulus margin $\tilde{M}_{m}$ for a given controller, defined as the shortest distance from the critical point -1 to all possible open-loop frequency responses obtained from the model set $G$, by varying $\Delta . \tilde{M}_{m}$ can then be written as:

$$
\begin{aligned}
& \tilde{M}_{m}=\inf _{\omega}\left(\inf _{\Delta} \mid 1+\right. K(j \omega) G_{n}(j \omega) \\
&(1+W(j \omega) \Delta(j \omega)) \mid)
\end{aligned}
$$

Eq. (19) expresses, that at every frequency, the minimal distance from the critical point to the uncertainty disk should be determined. Then the minimum of these distances with respect to the frequency gives $\tilde{M}_{m}$. Fig. 3 depicts the Nyquist plot of a nominal open-loop system with uncertainty disks represented for two different frequencies. It can easily be seen that, for a given frequency, the minimal distance from the critical point -1 to the corresponding disk is equal to the distance from the critical point to the frequency response of the nominal system, minus the radius of the disk. Hence:

$$
\begin{aligned}
& \inf _{\Delta}\left|1+K(j \omega) G_{n}(j \omega)(1+W(j \omega) \Delta(j \omega))\right| \\
&=\mid 1+ K(j \omega) G_{n}(j \omega) \mid- \\
&\left|K(j \omega) G_{n}(j \omega) W(j \omega)\right|
\end{aligned}
$$

$\tilde{M}_{m}$ is then given by: 
Table 1. Simulation results

\begin{tabular}{llcccccccc}
\hline Process & Method & $K_{p}$ & $T_{i}$ & $T_{d}$ & $M_{m}$ & $M_{c}$ & $\omega_{c}$ & $o$ & $t_{s}$ \\
\hline \multirow{3}{*}{$G_{p 1}$} & Ziegler-Nichols & 4.11 & 1.22 & 0.303 & 0.47 & 0.552 & 1.87 & 41.4 & 5.58 \\
& Kappa-Tau & 2.11 & 1.45 & 0.369 & 0.70 & 0.869 & 1.11 & 13.3 & 6.08 \\
& Proposed & 2.17 & 1.68 & 0.41 & 0.714 & 0.961 & 1.12 & 5.94 & 4.66 \\
& Ziegler-Nichols & 20.7 & 0.539 & 0.135 & 0.344 & 0.324 & 4.59 & 61.5 & 5.25 \\
$G_{p 2}$ & Kappa-Tau & 11.27 & 0.781 & 0.180 & 0.543 & 0.505 & 3.36 & 42.9 & 4.4 \\
& Proposed & 10.18 & 1.89 & 0.473 & 0.719 & 0.97 & 5.12 & 3.7 & 1.12
\end{tabular}

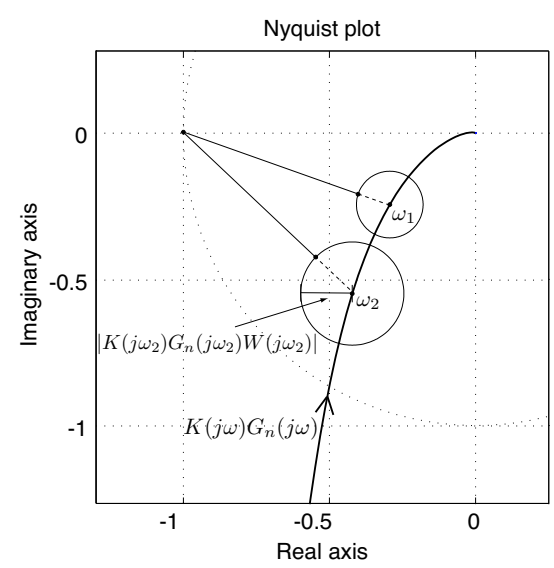

Fig. 3. Nyquist diagram of a nominal open-loop system with uncertainty disks represented at two frequencies

$$
\begin{aligned}
\tilde{M}_{m}=\inf _{\omega}(\mid 1+ & K(j \omega) G_{n}(j \omega) \mid \\
& \left.-\left|K(j \omega) G_{n}(j \omega) W(j \omega)\right|\right)
\end{aligned}
$$

and can easily be computed numerically.

In the frequency criterion of Eq. (2), $M_{m}$ should now be replaced by $\tilde{M}_{m}$ to consider the robustness against plant uncertainty. The derivative of $\tilde{M}_{m}$ with respect to the controller parameters is required to compute the gradient and Hessian of the frequency criterion:

$$
\frac{d \tilde{M}_{m}}{d \rho}=\frac{\partial \tilde{M}_{m}}{\partial \rho}+\frac{\partial \tilde{M}_{m}}{\partial \omega} \frac{\partial \tilde{\omega}_{M_{m}}}{\partial \rho}
$$

where $\tilde{\omega}_{M_{m}}$ is the frequency on the open-loop frequency response, which is determing for $\tilde{M}_{m}$. Under the assumptions that $\left|G_{n}(j \omega)\right|,|K(j \omega)|$, $|W(j \omega)|$ and their derivatives with respect to the frequency are continuous functions, one has: $\frac{\partial \tilde{M}_{m}}{\partial \omega}=0$, since $\tilde{M}_{m}$ is defined as the minimum of a function of $\omega$. Consequently:

$$
\begin{aligned}
& \frac{d \tilde{M}_{m}}{d \rho}=\frac{\partial\left|1+K\left(j \tilde{\omega}_{M_{m}}\right) G_{n}\left(j \tilde{\omega}_{M_{m}}\right)\right|}{\partial \rho} \\
& -\frac{\partial\left|K\left(j \tilde{\omega}_{M_{m}}\right) G_{n}\left(j \tilde{\omega}_{M_{m}}\right) W\left(j \tilde{\omega}_{M_{m}}\right)\right|}{\partial \rho}
\end{aligned}
$$

This derivatives can be computed analytically without any derivatives neither of the nominal plant model nor of the uncertainty profile, This information is interesting in a practical way, since uncertainty profiles are often available in the form of non-parametric models, and their derivatives with respect of the frequency are not directly known and can only be roughly approximated.

Other uncertainty models can be treated in a similar way. Approaches to get weighting functions in practice are treated in Doyle et al. (1992) with some examples.

\section{CONCLUSION}

An iterative method for designing the controller parameters with specifications on the modulus and complementary modulus margins as well as the crossover frequency has been proposed. A frequency criterion, defined as the weighted sum of squared errors between the desired and computed values of the design parameters, is minimized iteratively using the Gauss-Newton algorithm. The approach is flexible relative to the available plant model and the chosen controller structure.In addition, unstructured disk-like uncertainty profiles can be taken into account in the frequency criterion in a very simple way, to guarantee the robustness against model uncertainties. Simulation results show that the tuning method produces effectively adapted controllers for industrial plants.

\section{ACKNOWLEDGEMENTS}

This research work is financially supported by the Swiss National Science Foundation under grant No. 2100-064931.01

\section{REFERENCES}

Doyle, J., B. Francis and A.Tannenbaum (1992). Feedback Control Theory. Macmillan Publishing Company, New York.

Garcia, D., A. Karimi and R. Longchamp (2003). Data-driven controller tuning based on a frequency criterion. In: CDC03. pp. 127-132.

Ho, W. K., C. C. Hang and L. S. Cao (1995). Tuning of PID controllers based on gain and phase margin specifications. Automatica 31(3), 497-502.

K. J. Aström and T. Hägglund (1995). PID Controllers: Theory, Design and Tuning. 2nd ed.. Instrument Society of America.

Karimi, A., D. Garcia and R. Longchamp (2003). PID controller tuning using bode's integrals. IEEE Transactions on Control Systems Technology 11(6), 812-821. 